\title{
Mortalidad relacionada con el consumo de alcohol y su impacto en la esperanza de vida de la población del Noroeste Argentino, 2011
}

\author{
Carola Leticia Bertone* \\ Víctor Eduardo Torres ${ }^{\star *}$ \\ Marcos Javier Andrada ${ }^{\star \star \star}$
}

En el mundo el 5,9\% de las muertes ocurridas en el 2012 se debieron al consumo nocivo de alcohol (WHO, 2014). En Argentina, durante el año 2008, 11.013 muertes fueron atribuidas al consumo de alcohol (3,6\% del total de muertes en la población) (ACOSTA; BERTONE; PELÁEZ, 2012). El consumo de alcohol per cápita de personas de 15 años y más en Argentina se mantuvo alrededor de 9,5 litros de alcohol puro (equivalente a 25 gramos de alcohol diario), considerando el promedio de los años 2003 a 2005 y 2008 a 2010 (WHO, 2014). Este trabajo, cuantitativo, transversal y descriptivo, se propone estudiar la mortalidad atribuible al consumo de alcohol en las provincias del Noroeste Argentino (NOA) en 2011 y cómo afecta la esperanza de vida de dichas poblaciones. Se aplica la metodología propuesta por el Centro de Control y Prevención de Enfermedades (CDC por sus siglas en inglés) (1990) -mortalidad atribuible al consumo de alcohol (MACA) y años de esperanza de vida perdidos (AEVP) (ARRIAGA, 1996)-. Se utilizan datos de la Dirección de Estadísticas e Información de Salud (estadísticas vitales) y del Instituto Nacional de Estadística y Censos de la República Argentina (INDEC) (EnPreCoSP 2011 y proyecciones de población). Con este trabajo se espera aportar información actualizada acerca de la mortalidad atribuible al consumo de alcohol, insumo importante en términos de salud pública para valorar la implementación de estrategias adecuadas.

Palabras clave: Mortalidad. Alcohol. Noroeste Argentino. Años de esperanza de vida perdidos.

\footnotetext{
*Universidad Nacional de La Rioja (UNLAR), Centro de Estudios e Innovacion Tecnológica (CENIIT), Instituto de Demografia, La Rioja, Argentina (carolabertone@gmail.com; http://orcid.org/0000-0001-9022-0688).

** Universidad Nacional de Córdoba (UNC), Consejo Nacional de Investigaciones Cientificas y Técnica (CONICET), Córdoba, Argentina (torresedu@gmail.com; http://orcid.org/0000-0001-7719-8981).

*** Universidad Nacional de La Rioja (UNLAR), Centro de Estudios e Innovacion Tecnológica (CENIIT), Consejo Nacional de Investigaciones Cientificas y Técnica (CONICET), La Rioja, Argentina (licmarcosandrada@yahoo.com.ar; http://orcid. org/0000-0002-4067-1496).
} 


\section{Introducción}

En el mundo fallecieron alrededor de 3,3 millones de personas durante el año 2014 a causa del consumo nocivo de alcohol, lo que representa el 5,9\% del total de las muertes. Este nivel de consumo de alcohol se considera la causa de más de doscientas enfermedades, como también de trastornos mentales y comportamentales; afecta a personas de edades tempranas y genera discapacidad, y representa incluso el $25 \%$ de las defunciones entre los 20 y 39 años de edad (WHO, 2014). La mayor parte de esas muertes se deben a las enfermedades cardiovasculares o gastrointestinales, a lesiones, principamente de intención no determinada, y al cáncer (WHO, 2014).

El consumo de alcohol causa una considerable carga de morbilidad en Latinoamérica y el Caribe: 5,3\% de las defunciones de ambos sexos en el año 2000 (REHM et al., 2006), un $68 \%$ mayor que el promedio mundial en 2002 , lo que significa la muerte de una persona cada dos minutos en la región (MONTEIRO, 2007).

En 2010, el consumo mundial total de alcohol per cápita fue de 6,2 litros de alcohol puro y la prevalencia de episodios graves de consumo de alcohol (en la población total de 15 años o más) de 7,5\%. Mientras tanto, en América, ${ }^{1}$ el consumo total de consumo de alcohol per cápita fue de 8,4 litros de alcohol puro y la prevalencia de episodios graves de consumo de alcohol en la población total de 15 años o más alcanzó el 13,7\% durante ese mismo año (WHO, 2014).

El consumo de alcohol per cápita de personas de 15 años y más en Argentina se mantuvo en alrededor de 9,3 litros de alcohol puro (que equivale a 33,1 gramos de alcohol puro por día en promedio) en 2010. Dicho consumo apenas fue superado por Chile, y es muy similar al que presenta Estados Unidos. Sin embargo, en el caso de Chile el consumo per cápita total en bebedores fue inferior al de Argentina, cuyos otros países limítrofes tienen menor consumo total y solo en bebedores (ver Gráfico 1).

Una investigación sobre consumo de sustancias psicoactivas hecha en Argentina en 2010 detectó una prevalencia de consumo de alcohol de 44,5\% (IC 95\%: 42,8-46,2\%) en el mes previo al momento de la encuesta Dicha prevalencia casi no ha variado entre 2005 y 2011, de acuerdo a lo relevado por las Encuestas Nacionales de Factores de Riesgo (2005 y 2009) y las Encuestas sobre Consumo de Sustancias Psicoactivas (2008 y 2011) en el país. Por su parte, la prevalencia de consumo episódico excesivo de alcohol en el año 2010 fue de $12,0 \%$ en la población general y de $20,4 \%$ en los usuarios de alcohol (WHO, 2014).

Con relación a las consecuencias del uso del alcohol en Argentina, en 2008 el 3,6\% (11.013 casos) de las defunciones fueron atribuibles al consumo del acohol. De ellas, el $73 \%$ se produjo en hombres, principalmente jóvenes, cuyos decesos fueron debidos

\footnotetext{
${ }^{1}$ Países que componen la región América (AMR) para la Organización Mundial de la Salud: Antigua y Barbuda, Argentina, Bahamas, Barbados, Belice, Bolivia, Brasil, Canadá, Chile, Colombia, Costa Rica, Cuba, Dominica, República Dominicana, Ecuador, El Salvador, Granada, Guatemala, Guyana, Haití, Honduras, Jamaica, México, Nicaragua, Panamá, Paraguay, Perú, Puerto Rico, Saint Kitts y Nevis, Santa Lucía, San Vicente y las Granadinas, Suriname, Trinidad y Tobago, Estados Unidos de Norteamérica, Uruguay, Venezuela.
} 
a causas externas de mortalidad (códigos V01-Y99 de la Clasificación Internacional de Enfermedades en su décima versión) que incluyen accidentes de transporte, lesiones autoinflingidas, ahogamientos, entre otras. La esperanza de vida, como indicador del nivel de la mortalidad, se vio afectada cuatro veces más en los hombres que en las mujeres (ACOSTA; BERTONE; PELÁEZ, 2012). Acosta, Bertone y Peláez (2012) afirman que en el NOA el $2,5 \%$ de las muertes se atribuyen al consumo del alcohol. Si bien es una región en la que aparentemente este problema de salud pública es menor que en otras regiones, se propone conocer cómo es su situación al interior de la región, reconociendo que agrupar los datos puede esconder desigualdades que deben ser atendidas.

\section{GRÁFICO 1}

Consumo de alcohol per cápita de personas con 15 años y proporción de episodios de borracheras en personas de 15 años y más y de 15 a 19 años

Países seleccionados - 2010

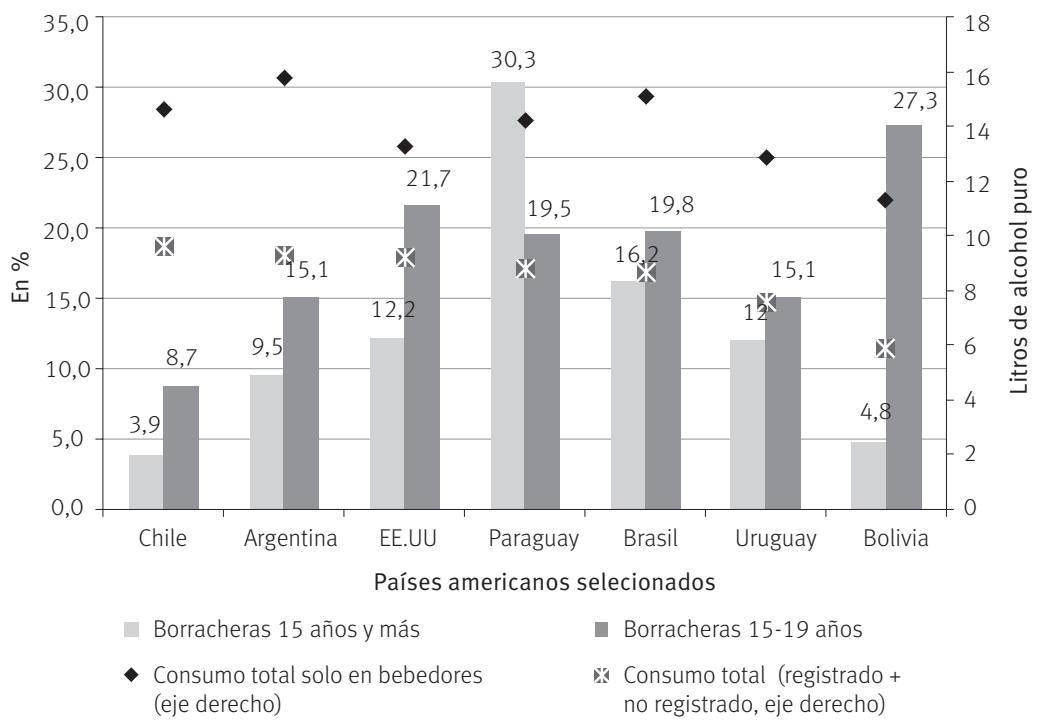

Fuente: WHO (2014)

Así, este trabajo se propone estudiar la mortalidad atribuible al consumo del alcohol en el NOA, dado que existen datos actualizados sobre la prevalencia del consumo de alcohol en Argentina (INDEC, 2011) y sobre la influencia de las causas de muerte relacionadas con dicho consumo (CDC, 2013). Por otra parte, y a diferencia de algunos trabajos antes mencionados, se aportará información para el ámbito provincial.

\section{Objetivo}

Este trabajo se propone estudiar la mortalidad atribuible al consumo de alcohol en cada una de las provincias que componen el NOA en 2011, valorando cómo afecta a la esperanza de vida en dichas poblaciones. 


\section{Noroeste argentino}

El NOA es un área geográfica compuesta por seis provincias: Catamarca, Jujuy, La Rioja, Salta, Santiago del Estero y Salta, las cuales representan, juntas, el 20,13\% de la superficie total del país. Según el Censo Nacional de Población, Hogares y Viviendas de 2010 , el $81,2 \%$ de la población era urbana, porcentaje que es inferior comparado con el resto del país, donde es $92,4 \%$. En el NOA, el 6,1\% de sus habitantes reside en zona rural agrupada y el $12,7 \%$ en la rural dispersa, mientras que en el país solo lo hacen el $2,9 \%$ y $4,7 \%$ respectivamente. En esta zona geográfica la agricultura es la actividad económica más importante. De acuerdo a los datos del mencionado censo la población total de esta región representaba el $12,2 \%$ del país y sus principales indicadores demográficos, comparados con el resto del país, se presentan en la Tabla 1.

TABLA 1

Indicadores demográficos seleccionados NOA y resto del país - 2010

\begin{tabular}{lrc}
\hline \multicolumn{1}{c}{ Indicador } & NOA & Resto del país \\
\hline Edad promedio (en años) & 29,53 & 32,97 \\
Edad mediana (en años) & 26 & 30 \\
Índice de masculinidad & 96,7 & 94,5 \\
Relación de dependencia & 37,2 & 35,5 \\
Porcentaje de inmigrantes internacionales & 1,5 & 4,9 \\
Porcentaje de inmigrantes nacionales & 10,1 & 21,4 \\
Porcentaje de población materno infantil & 42,2 & 39,5 \\
(menores de 6 años y mujeres de 10 a 49 años) & 50,8 & 51,4 \\
Porcentaje de población femenina & 44,2 & 34,9 \\
Porcentaje de población sin cobertura médica & 29,7 & 24,9 \\
Porcentaje de población de 0 a 14 años & 62,8 & 64,5 \\
Porcentaje de población de 15 a 64 años & 7,5 & 12,6 \\
Porcentaje de población de 65 años y más & 1,60 & 2,60 \\
Porcentaje de población de 80 años y más & 29,4 & 35,3 \\
Porcentaje de población con Secundario o & & \\
Polimodal completo o más &
\end{tabular}

Fuente: Censo Nacional de Población, Hogares y Viviendas ( INDEC, 2012).

Otro aspecto importante es que en el NOA hay mayor porcentaje de población sin cobertura médica respecto al resto del país, lo que señala que todavía hoy hay dificultades importantes en ese sentido. De igual modo, otro indicador que refleja que el NOA es una zona desfavorecida es el porcentaje de hogares con al menos una necesidad básica insatisfecha (NBI): según el censo nacional de 2010, el 15,6\% de los hogares del NOA estaba en esta categoría, mientras que en el resto del país lo era el $8,4 \%$.

\section{Legislación vigente sobre consumo del alcohol en Argentina y el NOA}

Argentina tiene una Ley Nacional de Lucha contra el Alcoholismo (24.788) desde 1997, que estipula entre sus artículos que lo siguiente está prohibido: la venta a menores de 
18 años, beber en la vía pública, las publicidades que incentiven el consumo de bebidas alcohólicas a menores de 18 años, en las que salgan menores o que no aclaren la edad mínima para beber y que se debe beber con moderación. También propone la creación de programas nacionales de prevención y de lucha contra el consumo excesivo de alcohol y la inclusión en todos los niveles educativos de contenidos curriculares sobre el consumo excesivo y sus patologías. Asimismo, dicha ley se ve complementada por la Ley 26.363 de 2008, Ley de Tránsito y Seguridad Vial, que regula cuestiones atinentes a la conducción bajo los efectos del alcohol.

En el año 2014, se promulgó el Plan Integral para el Abordaje de los Consumos Problemáticos (Ley Nacional 26.934), cuyos objetivos contemplan aspectos preventivos, asistenciales y de integración y amparo de sujetos con algún tipo de consumo problemático que afecta negativamente, en forma crónica la salud física o psíquica del sujeto o sus relaciones sociales. Se obliga en esta norma a las provincias a prestar asistencia gratuita a las personas afectadas por este tipo de problema.

En el ámbito provincial, La Rioja, Tucumán, Jujuy y Catamarca son las jurisdicciones en las que se visualizan programas que buscan atender de manera preventiva y asistencial el consumo de alcohol y donde, desde políticas bio-psicosociales, se abordan los trastornos relacionados con el alcohol.

La Ley Nacional 26.363 (2008) prohíbe conducir bajo los efectos del alcohol y establece como límite para hacerlo los $500 \mathrm{mg}$ /litro de sangre para conductores particulares y cero para vehículos destinados al transporte de pasajeros, de menores y de carga. A esta ley nacional adhieren todas las provincias del NOA, que, a su vez, han promulgado leyes propias sobre el consumo de alcohol. Por ejemplo, Salta, Tucumán, La Rioja y Catamarca establecen dentro del marco alcoholemia cero que los conductores no deben poseer ningún tipo de graduación alcohólica en sangre. Las sanciones son diversas y consideran desde la retención del vehículo y multas hasta treinta días de arresto. Las últimas en adherir a este tipo de medidas fueron Salta y Tucumán. Jujuy, en una ley promulgada en 2012, estipula lugares y horarios de venta, también contemplados en algunas leyes provinciales del NOA.

Se puede afirmar que Catamarca, La Rioja y Tucumán son las provincias con leyes más rigurosas en relación con el consumo y la venta de bebidas alcohólicas. Se agrega a ellas Salta, en una segunda instancia, con la Ley Alcoholemia Cero o Tolerancia Cero a partir de 2014, y, por último, Santiago del Estero, donde, además de la adhesión parcial a la Ley Nacional, solo se ha constatado el decreto n. 56 del año 2000, que regula la venta de bebidas alcohólicas.

\section{Materiales y métodos}

El presente estudio es de tipo cuantitativo, transversal y descriptivo, cuyo universo de estudio está conformado por las defunciones producidas en 2011 (se utiliza el promedio de defunciones producidas entre los años 2010 y 2012) en cada provincia. Se estudian dichas defunciones según causas de muerte atribuibles, directa e indirectamente, al consumo de 
alcohol. Se toma como base la metodología propuesta por el Centro de Control y Prevención de Enfermedades (CDC, por sus siglas en inglés) (CDC, 2013) mediante el cual se estima la mortalidad atribuible al consumo de alcohol (MACA). Posteriormente, para valorar el impacto sobre la esperanza de vida al nacer, calculan los años de esperanza de vida perdidos (AEVP) (ARRIAGA, 1996).

Los datos se extraen de las bases de datos de defunciones de la Dirección de Estadísticas e Información en Salud (DEIS) del Ministerio de Salud de la Nación entre los años 2010 y 2012, y las proyecciones de población del Instituto Nacional de Estadísticas y Censos (INDEC) calculadas para 2011 de cada provincia del NOA. También se utilizaron tablas de vida de cada provincia realizadas por el INDEC para el período 2008-2010, insumo necesario para la estimación de los AEVP, y la Encuesta Nacional sobre Prevalencia de Consumo de Sustancias Psicoactivas del año 2011 (EnPreCoSP-2011), elaborada por el INDEC, para la estimación de la prevalencia del consumo de alcohol.

Es apropiado mencionar algunas características respecto a la calidad de las fuentes de datos que se utilizan en este trabajo. En primer lugar, el porcentaje de cobertura del registro de las muertes, según reportes nacionales, alcanza el 99\% en Argentina (NACIONES UNIDAS, 2008). Con relación a la declaración de la causa de defunción, un estudio que evaluó los errores en la información sobre la causa básica de muerte, a través del porcentaje de "causas mal definidas", asegura que Argentina presenta un nivel aceptable en este rubro (8,1\% del total de defunciones entre 2011 y 2013). Sin embargo, otros trabajos comentan que la exactitud de los registros de defunción de Argentina está comprometida, ya que el porcentaje de causas poco útiles, indicador utilizado actualmente, es elevado (39\%). Así, Argentina presenta la coexistencia de una elevada cobertura del registro de defunciones con un porcentaje muy alto de causas de muerte "poco útiles" (RIBOTTA, 2014). Reconociendo esto, y dado que no se hallaron estudios regionales al interior del país, se estimó el porcentaje de códigos poco útiles, de acuerdo a Naghavi y otros (NAGHAVI et al., 2010) para cada región de Argentina para reconocer las limitaciones de este estudio en ese sentido. Se utilizó el total de muertes del país, incluyendo todas las edades y las causas de defunciones, ordenadas por región de residencia de los difuntos de 2011. La región del NOA es la tercera en orden decreciente con una proporción de código poco útiles de $36 \%$ dentro de un rango que varía desde $23 \%$ hasta $44 \%$.

Cabe destacar que algunos códigos poco útiles incluyen aquellos que corresponden a enfermedades hipertensivas (I10-I15) y otros que corresponden a patologías del aparato digestivo que el CDC considera como causas atribuibles al consumo del alcohol. Sin embargo, en los años en los que se enfoca este estudio, las enfermedades hipertensivas y digestivas incluidas en los códigos poco útiles puestos en discusión, y que se incluyen en el estudio de la MACA, representaron en 2011 solo el 4,5\% del total de defunciones, de manera tal que aquel porcentaje de causas poco útiles según las categorías del autor elegido para realizar la estimación se vería reducido. Sin embargo, es un hecho que mejorar la declaración de la causa de muerte podría modificar los resultados de este estudio, que quizás esté subestimando el número de muertes atribuibles al alcohol. 
Para la estimación de la MACA se ordenaron las defunciones según las muertes hayan sido por causas directamente relacionadas con el consumo de alcohol (CDCA) o por causas indirectamente relacionadas con el consumo de alcohol (CICA), tanto agudas como crónicas. Las CDCA son por definición atribuibles al consumo de alcohol y se consideran como $100 \%$ atribuibles, por lo cual presentan una fracción atribuible poblacional (FAP) ${ }^{2}$ de 1,00 . Por su parte, en el caso de las CICA se precisa calcular la FAP, la cual requiere tener la prevalencia de dicho consumo en la población para la que se hace el análisis (ver más adelante).

El cálculo de la FAP:

$\mathrm{FAP}=\left[\frac{\mathrm{r}_{1}-1}{\left(r_{1}-1\right)+1 / \mathrm{p}_{1 \%}}\right]+\left[\frac{\mathrm{r}_{2}-1}{\left(r_{2}-1\right)+1 / \mathrm{p}_{2 \%}}\right]$

Donde:

$\mathrm{r}_{1}=$ cociente entre el riesgo relativo medio y bajo (CDC, 2013);

$r_{2}=$ cociente entre el riesgo relativo alto y bajo (CDC, 2013);

$\mathrm{p}_{1}=$ prevalencia de consumo de alcohol rango medio (INDEC, 2011);

$\mathrm{p}_{2}=$ prevalencia de consumo de alcohol rango alto (INDEC, 2011).

Estos cálculos utilizan estimaciones de riesgo agrupadas, que fueron obtenidas a partir de metaanálisis, sobre la relación entre el alcohol y diversas causas, así como datos sobre la prevalencia del consumo de alcohol en niveles específicos. La mayoría de los cálculos de riesgo utilizados en este método se obtuvieron de un estudio realizado por English et al. (1995) con algunas causas de muerte extraídas de otros metaanálisis (CDC, 2013). A los efectos de este trabajo, se asume que esta región de Argentina presenta tales riesgos relativos.

La prevalencia del consumo de alcohol se extrajo de la EnPreCoSP 2011, de la cual se han tomado algunas variables del bloque Bebidas Alcohólicas de esa encuesta, los cuales permitieron el cálculo del consumo medio diario de alcohol:

$\mathrm{CMD}=\left[\left(\mathrm{Q}^{\star} \mathrm{AF}\right)+\left(\mathrm{BF}{ }^{\star} \mathrm{BQ}\right)\right]$

Donde:

CMD es el consumo medio diario de alcohol;

Q es el número promedio de tragos por día;

AF es la frecuencia ajustada de consumo de alcohol: número de días en los que se consume alcohol menos el número de borracheras;

BF es el número de borracheras;

BQ es la cantidad de tragos consumidos en una borrachera.

Cabe destacar que para el cálculo de la cantidad de tragos consumidos en la borrachera (BQ), la encuesta no cuenta con una variable que dé cuenta del número de tragos consumidos en un evento de borrachera. Sin embargo, existe la variable categórica sobre

\footnotetext{
${ }^{2}$ FAP: es la reducción proporcional de los enfermos o de los muertos que debería ocurrir si la exposición al factor de riesgo fuera reducida a un escenario alternativo e ideal de exposición
} 
si tuvo un consumo excesivo de cerveza, vino o bebidas fuertes. Ante la respuesta positiva de esa variable se asume que al menos ha consumido ocho tragos de cerveza, cinco de vino o tres de bebidas fuertes, tal como se define el consumo excesivo en la CDC, de modo que se trataría del número mínimo de tragos en la borrachera, con lo cual los valores del consumo medio diario todavía podrían ser superiores.

Así, para determinar el tipo de consumo, se utilizan los puntos de corte que se detallan en Cuadro 1.

CUADRO 1

Puntos de corte utilizados para establecer nivel de consumo

\begin{tabular}{|ccc|}
\hline Consumo & Hombres & Mujeres \\
\hline Bajo & Hasta 3,0 tragos por día & Hasta 1,5 tragos por día \\
Medio & 3,1 tragos por día & 1,6 tragos por día \\
Alto & 4,5 tragos por día & 3,0 tragos por día \\
\hline
\end{tabular}

Fuente: English et al. (1995); Ridolfo y Stevenson (2001).

Para calcular las muertes atribuibles al alcohol (MACA), la FAP para cada causa de defunción indirectamente atribuible al alcohol se multiplica por el número de muertes observadas en una categoría de edad determinada (CDC, 2013).

Posteriormente se estimaron los AEVP para cada una de las provincias del NOA, con el objetivo de valorar el impacto de las MACA en la esperanza de vida al nacer de esas poblaciones.

El indicador AEVP está basado en la estimación de las esperanzas de vida temporarias, que miden el promedio de años de vida que una población de edad exacta x vive desde dicha edad $x$ hasta otra de $x+n$ años posterior. Por ejemplo, si la esperanza de vida temporaria entre las edades 40 y 65 años fuera de 19 años, significa que la población que cumple 40 años va a vivir en promedio 19 años (debido a la mortalidad de algunos de los integrantes del grupo). Por lo tanto, si esa población vive en promedio 19 años del máximo de 25, significa que en promedio se pierden 6 años de esperanza de vida.

De este modo, la definición de AEVP es:

${ }_{\mathrm{n}} \mathrm{AEVP}_{\mathrm{x}}=\mathrm{n}-{ }_{\mathrm{n}} \mathrm{e}_{\mathrm{x}}$

Donde $n$ es el intervalo entre las dos edades consideradas. De acuerdo con lo que indica el propio Arriaga, “cuando se analizan causas de muerte este índice es el más adecuado para medir el nivel y cambio de la mortalidad de cada causa de muerte" (ARRIAGA, 2012, p. 101). En este caso se utilizó como el último grupo de edad al de 80 años y más. De este modo, "con base en las tasas específicas de mortalidad por edades, se calcula la vida promedio que la población vive (esperanza de vida) y se determina cuántos años de vida se pierden considerando la hipótesis sobre cuántos años debería vivir" (ARRIAGA, 1996, p. 9). De esta manera, el indicador presenta dos ventajas principales: no se ve afectado por la estructura de edades de la población y su resultado se mide en años, lo cual facilita la interpretación y comparación entre poblaciones. 


\section{Resultados}

Antes de comenzar el análisis es pertinente mencionar que este trabajo presenta algunas limitaciones devenidas de las fuentes de datos utilizadas. Entre ellas, se destaca la deficiente declaración de causa de muerte del registro de defunciones, a partir de la cual los resultados las muertes atribuibles al consumo del alcohol podrían ser mayores. Asimismo, hay que recordar que la prevalencia del consumo del alcohol podría ser mayor a la estimada a partir de la fuente disponible para este fin. Con ello, podría significar que los hallazgos de este estudio sobre el nivel de la MACA podrían ser superiores a los detectados, e incluso las diferencias halladas entre las provincias del NOA ser distintas si dichas limitaciones no existieran.

La mortalidad atribuible al consumo de alcohol en todas las edades en el NOA durante 2011 ronda el $9 \%$ en hombres y el 3,5\% en mujeres respecto al total de defunciones de cada grupo. Sin embargo, se observan diferencias entre las provincias que componen la región: Catamarca, Jujuy y Salta están por encima del 10\% de defunciones masculinas debidas al consumo de alcohol mientras que en los óbitos femeninos sobresalen Jujuy y Salta. Esta última provincia presenta en ambos sexos un porcentaje de muertes debido a este factor de riesgo superior al promedio de las provincias del NOA en conjunto. Por otra parte, en el Gráfico 2 puede observarse el porcentaje de población con consumo alto y medio que, en el caso de hombres ronda -entre ambas categorías- el 61\% (excepto Tucumán), mientras que en mujeres la dispersión es mayor: Salta, con 16\%, registra el menor valor y el máximo se encuentra en $30 \%$ en Santiago del Estero y Tucumán.

\section{GRÁFICO 2}

Proporción de población según consumo medio y alto, por sexo y provincia de residencia NOA - 2011

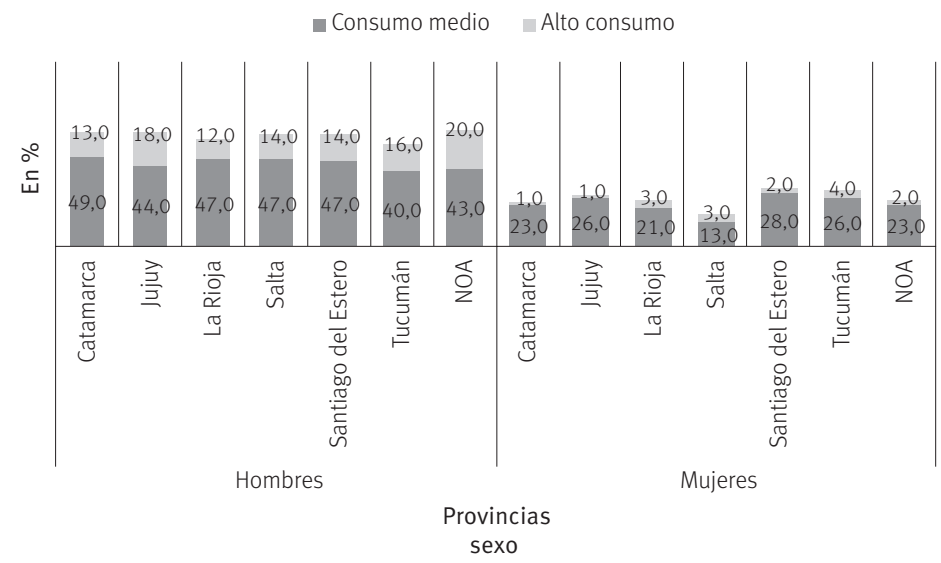

Fuente: INDEC (2011).

En la Tabla 2 se exponen las defunciones del NOA durante 2011, las tasas de mortalidad (por cada 100.000 habitantes y ajustadas por edad), sus respectivos errores estándares 
relativos y la razón entre las causas atribuibles y las no atribuibles al consumo del alcohol. Los resultados señalan que las tasas de mortalidad en hombres por causas atribuibles al consumo de alcohol se encuentran entre 34 y 67 defunciones cada 100.000 habitantes masculinos, mientras que las tasas femeninas se encuentran entre 13 y 21 óbitos por cada 100.000 mujeres residentes en las provincias del NOA. La Rioja y Santiago del Estero presentan las tasas más altas de mortalidad por causas atribuibles al consumo de alcohol del NOA, mientras que Jujuy y Salta son las provincias la región donde la población de mujeres tiene el mayor riesgo de morir de enfermedades atribuibles al consumo de alcohol.

Adicionalmente, si se realiza el cociente entre 100 y la razón de tasas de cada provincia (y de la región) presentada en la tabla 3, se obtiene la cantidad de personas que fallecen por enfermedades atribuibles al consumo de alcohol cada 100 fallecidos por causas no atribuibles al alcohol. Utilizando dicha lógica de análisis, puede destacarse que en la región mueren alrededor de 10 hombres por enfermedades atribuibles al consumo de alcohol de cada 100 fallecidos por causas no atribuibles al alcohol. Esta última relación de fallecimientos encuentra su valor más bajo en Santiago del Estero y La Rioja (alrededor de 7) mientras que en Salta y Jujuy es al contrario (en torno a 13).

TABLA 2

Defunciones, tasas de mortalidad y razón de tasas por causas atribuibles y no atribuibles al consumo de alcohol por provincia

NOA - 2011

\begin{tabular}{|c|c|c|c|c|c|c|c|c|c|}
\hline Provincias & Población & MNACA & $T_{M N A C A}{ }^{a}$ & TMNACAA $^{\mathrm{b}}$ & MACA & $\mathrm{TMACA}^{\mathrm{a}}$ & TMACAA $^{\mathrm{b}}$ & $\begin{array}{c}\text { Razón } \\
\text { de tasas }\end{array}$ & $\mathrm{e}_{0}$ \\
\hline \multicolumn{10}{|c|}{ Hombres } \\
\hline Catamarca & 206.929 & 838 & 405 & 417,8 & 100 & 48,5 & 50,7 & 12 & 73,3 \\
\hline Jujuy & 351.266 & 1.728 & 492 & 517,5 & 235 & 66,9 & 70,5 & 13,6 & 71,8 \\
\hline La Rioja & 182.266 & 845 & 463,8 & 506,9 & 63 & 34,5 & 35,8 & 7,4 & 72,3 \\
\hline Salta & 641.628 & 2.933 & 457,1 & 500,5 & 376 & 58,6 & 62,5 & 12,8 & 71,7 \\
\hline $\begin{array}{l}\text { Santiago del } \\
\text { Estero }\end{array}$ & 451.272 & 2.266 & 502,1 & 521,2 & 153 & 33,9 & 34,2 & 6,8 & 70,9 \\
\hline Tucumán & 757.874 & 3.871 & 510,8 & 488,9 & 312 & 41,2 & 40,8 & 8,1 & 72,2 \\
\hline NOA & 2.591 .235 & 12.481 & 481,7 & 488,9 & 1.239 & 47,8 & 48,6 & 9,9 & $-\cdots-$ \\
\hline \multicolumn{10}{|c|}{ Mujeres } \\
\hline Catamarca & 205.307 & 854 & 415,8 & 312,6 & 26 & 12,9 & 11,7 & 3,1 & 78,8 \\
\hline Jujuy & 356.438 & 1.503 & 421,6 & 358,8 & 76 & 21,5 & 20,0 & 5,1 & 78,1 \\
\hline La Rioja & 180.236 & 727 & 403,4 & 352,3 & 26 & 10 & 9,7 & 2,5 & 78,6 \\
\hline Salta & 647.644 & 2.483 & 383,4 & 347,0 & 111 & 17,2 & 16,6 & 4,5 & 78,2 \\
\hline $\begin{array}{l}\text { Santiago del } \\
\text { Estero }\end{array}$ & 441.466 & 1.866 & 422,6 & 352,7 & 58 & 13,1 & 11,7 & 3,1 & 77,8 \\
\hline Tucumán & 771.651 & 3.667 & 475,2 & 338,8 & 132 & 17,1 & 14,8 & 3,6 & 78,2 \\
\hline NOA & 2.602 .742 & 11.099 & 426,4 & 344,6 & 421 & 16,2 & 14,8 & 3,8 & $-\cdots$ \\
\hline
\end{tabular}

Fuente: Argentina (2011, 2012, 2013); INDEC (2011, 2013).

a Tasas expresadas por cada 100.000 habitantes; b: Tasas ajustadas por edad (método directo-población mundial); MNACA: defunciones no atribuibles al consumo de alcohol; TMNACA: tasas brutas de mortalidad no atribuibles al consumo de alcohol; MACA: defunciones atribuibles al consumo de alcohol; TMACA: tasas brutas de mortalidad atribuibles al consumo de alcohol; $\mathrm{e}_{0}$ : esperanza de vida al nacer $2008-2010$. 
Como se comentara anteriormente, las mujeres se ven menos afectadas por estados mórbidos relacionados con el consumo del alcohol: continuando con la línea de análisis precedente se percibe que en la región fallecen por esas causas casi 4 mujeres por cada 100 decesos femeninos no vinculados al alcohol, 3 de cada 100 en Jujuy y 4,5 de cada 100 en Salta, lo que evidencia que además de ser un efecto menor también el rango de variación en el caso de las mujeres en considerablemente menor que en los hombres.

Asimismo, en la Tabla 2 se presentan las tasas estandarizadas de las causas atribuibles y no atribuibles: al analizar el cambio porcentual entre las tasas brutas y las estandarizadas se percibe, en el caso de los hombres, que en todas las provincias la estructura de la población provoca un efecto similar, excepto en Tucumán, donde es mayor. Por su parte, en el caso de mujeres, en Catamarca y Tucumán es donde el efecto edad es más fuerte. En el próximo apartado se presenta cómo afecta esta mortalidad a la esperanza de vida de las personas a partir del indicador AEVP.

\section{AEVP por causas atribuibles al consumo de alcohol}

En el caso de hombres, en el NOA se perdieron aproximadamente 11 años de esperanza de vida por todas las causas de muertes, de los cuales un promedio de 0,8 años se debe a causas atribuibles al consumo de alcohol. Catamarca y Tucumán perdieron algunos años menos (9,7 y 10,4 respectivamente). Según se percibe en el gráfico 3, los AEVP de causas no atribuibles al consumo de alcohol van dese 9, en Catamarca, hasta 10,8, en Santiago del Estero. Por su parte, aquellos atribuibles al consumo de alcohol han generado en la región entre 0,5 y 1,3 AEVP, en La Rioja y Jujuy respectivamente.

\section{GRÁFICO 3}

AEVP según causas atribuibles al consumo de alcohol en hombres, por provincias NOA - 2011

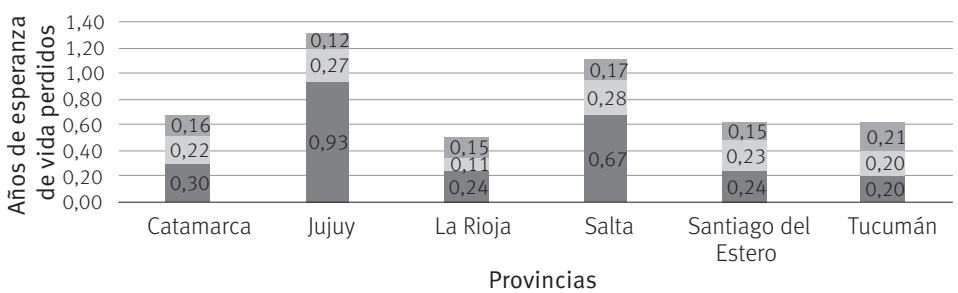

Causas de muerte indirectamente relacionadas con el consumo de alcohol

Causas de muerte directamente relacionadas con alcohol agudas

Causas de muerte directamente relacionadas con alcohol crónicas

Fuente: Argentina (2011, 2012, 2013); INDEC (2011).

Sin embargo, es importante tener en cuenta la calidad de las fuentes de datos. Para valorar la calidad de la declaración de las causas de muerte, pertinente en este caso, se utiliza como indicador el porcentaje de causas mal definidas (BAY; ORELLANA, 2007). Al respecto, en una investigación que valoró dicho porcentaje entre los años 2000 y 2010 en Argentina, 
se asegura que no se observa un comportamiento que refleje mejoras, sino que, por el contrario, dicho porcentaje alcanzó el 8,4\% en 2010. A su vez, ese trabajo estudió el indicador por jurisdicciones en todo ese período, en el que se observó que Jujuy, Santiago del Estero y Tucumán presentaron un porcentaje de causas de muerte mal definidas superior al $10 \%$ (ver figura 8 en BERTONE, 2014). De este modo, cabe preguntarse cómo influye la calidad del registro de defunciones en relación con la mala declaración de la causa de muerte en las diferencias encontradas entre las provincias de la región. Sin embargo, esta pregunta no será respondida en este trabajo, pero debe ser tenida en cuenta a la hora de las conclusiones.

Como se explicara anteriormente, existen tres tipos de causas de muerte en relación con el consumo de alcohol: las causas directamente relacionadas con el consumo de alcohol (CDCA) agudas, las CDCA crónicas y las causas indirectamente relacionadas con el consumo del alcohol (CICA). Al observar la mortalidad en relación con dichas categorías, las causas directamente vinculadas provocan más AEVP respecto a las indirectas. A su vez, dentro de las CDCA, las crónicas generan mayores AEVP que las agudas.

Las CDCA crónicas triplican el número de AEVP que provocan las CICA agudas en Jujuy y lo duplican en Salta. Además, si se pone el foco en las CDCA crónicas por grupo de edades, se destaca cómo los AEVP se disparan a temprana edad en Salta y Jujuy, describiendo dos picos: uno alrededor de los 40 años y otro pasados los 50. Recordando que los AEVP darán más peso a muertes prematuras y a gran número de casos en edades más tardías, he aquí parte de la explicación de por qué este grupo de causas genera la mayor pérdida en la esperanza de vida de estas poblaciones. En el resto de las provincias se aumentan progresivamente los AEVP a partir de los 35 años, describe una meseta y disminuye hacia los 70 años (Gráfico 4).

GRÁFICO 4

AEVP según causas directamente relacionadas con el consumo de alcohol en hombres, por provincias NOA - 2011
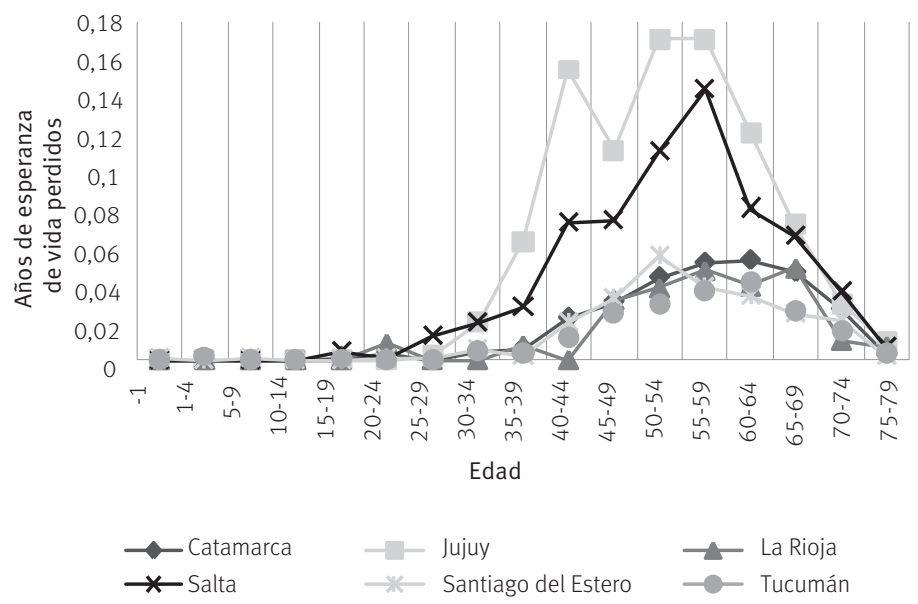

Fuente: Argentina (2011, 2012, 2013); INDEC (2011). 
Al indagar sobre qué causas provocaban los comentados picos en Jujuy y Salta se pudo detectar que, en su mayor parte, se deben a la enfermedad alcohólica del hígado (K70) en las muertes más tempranas y a cirrosis hepática (K74 y K76) y dependencia al alcohol (F10.2) en el segundo pico. Cabe destacar que la cirrosis hepática es la fase final de la enfermedad alcohólica del hígado y ambos cuadros mórbidos ocurren después de años del abuso prolongado del alcohol.

Se podría hipotetizar que en estas dos provincias, y bajo el supuesto que biológicamente los hombres de estas poblaciones están en iguales condiciones de susceptibilidad de enfermar ante el consumo de alcohol, podría decirse que existe un mayor problema de salud pública por el uso nocivo del alcohol respecto al resto de la región.

Por otra parte, la mortalidad femenina genera alrededor de 7 AEVP, presentando el valor más bajo en Catamarca y el mayor en Santiago del Estero. Como era de esperar, la mortalidad en las mujeres es menor. Jujuy y Tucumán son las provincias donde se estimaron los AEVP por causas atribuibles al consumo de alcohol más altos del NOA, donde el promedio de AEVP es de 0,2 .

En términos generales, y a diferencia de los hombres, las CICA generan casi tantos AEVP como las directas, e incluso en algunas provincias provocan más AEVP, como en el caso de Tucumán, La Rioja y Catamarca. Por su parte, en Jujuy las CDCA crónicas generan la mayor parte de los AEVP.

\section{GRÁFICO 5}

AEPV según causas atribuibles al consumo de alcohol en mujeres, por provincias NOA - 2011

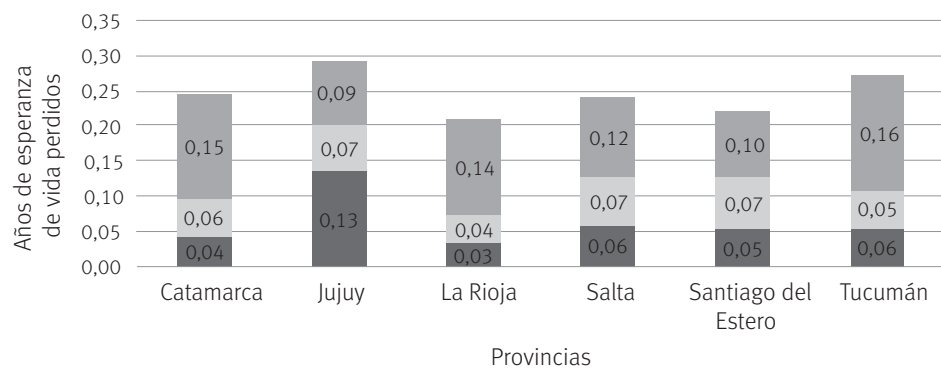

Causas de muerte indirectamente relacionadas con el consumo de alcohol

Causas de muerte directamente relacionadas con alcohol agudas

- Causas de muerte directamente relacionadas con alcohol crónicas

Fuente: Argentina (2011, 2012, 2013); INDEC (2011).

Con el fin de despejar las incógnitas que se presentaron a raíz del Gráfico 6, se presentan a continuación los AEVP por CDCA crónicas por grupo de edad en las mujeres. A través de los resultados se aprecia un comportamiento similar al de los hombres, con la presencia de dos picos: uno alrededor de los 30 años de edad y otro a partir de los 50. De igual modo que en el caso masculino, las causas que más aportan casos a este grupo de causas son la enfermedad alcohólica del hígado y la cirrosis hepática. 
Ahora bien, en Tucumán, La Rioja y Catamarca, las CICA que aumentan los AEVP en dichas provincias son: accidente cerebrovascular agudo hemorrágico (160-162, 169.0-169.2) en Catamarca, enfermedad cardíaca isquémica (120-125) en Tucumán e hipertensión arterial (I10-I15) en La Rioja.

\section{GRÁFICO 6}

AEVP según causas directamente relacionadas con el consumo de alcohol en mujeres, por provincias NOA - 2011

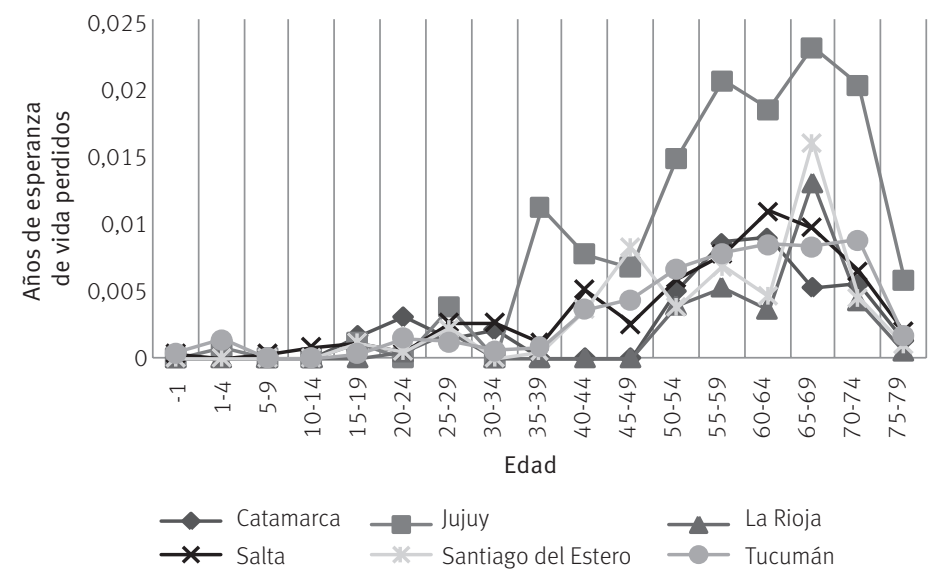

Fuente: Argentina (2011, 2012, 2013); INDEC (2011),

\section{Consideraciones finales}

En 2011, el NOA presentó una proporción de mortalidad atribuible al alcohol 2\% superior a la mundial en hombres y $0,5 \%$ menor en las defunciones de mujeres (WHO, 2014) del año 2012, y son superiores a las halladas en otros estudios realizados en el país con datos de 2008 (ACOSTA; BERTONE; PELÁEZ, 2012).

Este trabajo aporta información actualizada acerca de la mortalidad atribuible al consumo de alcohol que resulta imprescindible para la aplicación de políticas públicas y para su seguimiento a partir de la normativa jurídica emanada para enfrentar este serio problema sanitario y social. La estimación de la mortalidad atribuible al consumo de alcohol en todas las edades en el NOA a partir de la metodología descripta por el CDC no es un dato menor aun cuando las pérdidas en las esperanzas de vida no son de tanta gravedad, como por ejemplo supone la mortalidad infantil, y lógicamente perfectible si se dispone de una fuente de datos con mejor calidad de información, de modo tal de que fuera factible incorporar al análisis aspectos que quedan ocultos pero que pueden provocar la subestimación de los efectos del consumo de alcohol.

La metodología adoptada a través de AEVP permite inferir que, efectivamente, aun cuando no es la causa que más contribuye a la pérdida de años esperanza de vida, el consumo de alcohol-que afecta principalmente a hombres- reduciría en un año la esperanza de vida de la región. El indicador AEVP tiene en cuenta la estructura etaria de la población, 
con lo cual se estaría considerando además la estructura poblacional de la región muy próxima a la media nacional.Como se mencionó, en toda la región se pierde casi un año de esperanza de vida a causa del consumo de alcohol en hombres, lo que afecta especialmente a jujeños y salteños, quienes sufren y mueren principalmente de patologías directamente relacionadas con el consumo de alcohol, como la enfermedad alcohólica del hígado y la cirrosis hepática. Estas muertes ocurren aproximadamente a partir de los 30 años, con un pico en los 40 y otro a los 60 años de edad.

Las mujeres se ven menos afectadas por estas causas de muerte, ya que pierden el equivalente a unos 2 meses de esperanza de vida por consumo de alcohol y se ven afectadas principalmente por las causas indirectamente atribuibles al consumo del alcohol. Sin embargo, Jujuy no solo es la jurisdicción con más AEVP por consumo de alcohol, sino que también las causas que más afectan a las jujeñas son las crónicas, tal como ocurre en los hombres. Ocurre algo opuesto en Tucumán y en Catamarca, donde son las causas indirectas las que más AEVP generan. Estas tres provincias son las que más años de esperanza pierden por el consumo de alcohol en mujeres.

En resumen, las provincias de Jujuy y Salta son las jurisdicciones del NOA más afectadas por este problema de salud pública. Esto no es exclusivo de la población masculina, por lo cual se puede hipotetizar sobre alguna influencia cultural que coloca a estas poblaciones en situaciones particulares en relación con el resto de las provincias de la región. Sin embargo esto no se refleja en los datos sobre prevalencia del alcohol de las diferentes encuestas realizadas en el país (INDEC, 2011; FERRANTE et al., 2011; SEDRONAR, 2010). Con ello, y tratándose de mortalidad, se podría decir que implicaría una deficiencia en la asistencia de las personas con consumos de riesgo en esas provincias.

Se espera que a partir de la promulgación del Plan Integral para el Abordaje de los Consumos Problemáticos (Ley Nacional 26.934/2014), cuyos objetivos contemplan aspectos preventivos, asistenciales y de integración y amparo de sujetos con algún tipo de consumo problemático, se atienda este problema en dichas jurisdicciones.

\section{Referencias}

ACOSTA, L.; BERTONE, C.; PELÁEZ, E. Mortalidad y Años de Esperanza de Vida Perdidos a causa del consumo de alcohol en Argentina. 2008. Población y Salud en Mesoamérica, v. 9, n. 2, p. 1-21, 2012.

ARGENTINA. Ministerio de Salud. Base de datos de mortalidad 2010. Buenos Aires: Dirección de Estadísticas e Información de Salud, 2011.

Base de datos de mortalidad 2011. Buenos Aires: Dirección de Estadísticas e Información de Salud, 2012.

. Base de datos de mortalidad 2012. Buenos Aires: Dirección de Estadísticas e Información de Salud, 2013.

ARRIAGA, E. Comentarios sobre algunos índices para medir el nivel y el cambio de la mortalidad. Estudios Demográficos y Urbanos, v. 11, n. 1, p. 5-30, 1996. 
. Analisis demográfico de la mortalidad. Córdoba: Universidad Nacional de Córdoba, 2012. . Los años de vida perdidos: su utilización para medir los niveles y cambios de la mortalidad. Notas de Población, Santiago de Chile, v. 24, n. 63, p. 7-38, Junio 1996.

BAY, G.; ORELLANA, H. La calidad de las estadísticas vitales en la América Latina. Santiago de Chile: CEPAL-UNFPA-OPS, Diciembre 2007.

BERTONE, C. L. Determinantes sociales de la mortalidad infantil en Argentina 2000-2010. Córdoba, 2014.

CDC - Centers for Disease Control and Prevention. Alcohol-related mortality and years of potential life lost--United States, 1987. MMWR Morb Mortal Wkly Rep, n. 39, p. 272-275, 1990.

. Alcohol Related Disease Impact (ARDI) application, 2013. Disponivel em: 〈https:// nccd.cdc.gov/DPH_ARDI/Info/Methods.aspx>. Acesso em: 20 mar. 2016.

ENGLISH, D.; HOLMAN, C.; MILNE, E.; WINTER, M.; HULSE, G.; CODDE, J.; KURINCZUK, J. The quantification of drug caused morbidity and mortality in Australia. Canberra: Australian Government Public Service, 1995.

FERRANTE, D.; LINETZKY, B.; KONFINO, J.; KING, A.; VIRGOLININ, M.; LASPIUR, S. Encuesta Nacional de Factores de Riesgo 2009: evolución de la epidemia de enfermedades crónicas no transmisibles en Argentina. Estudio de corte transversal. Revista Argentina de Salud Pública, Buenos Aires, v. 2, n. 6, p. 34-41, 2011.

INDEC - Instituto Nacional de Estadística y Censos. Encuesta Nacional sobre Prevalencias de Consumo de Sustancias Psicoactivas (ENPreCoSP). Buenos Aires, 2011.

. Tablas abreviadas de mortalidad por sexo y edad 2008-2010. 1. ed. Buenos Aires, 2013. Disponivel em: $<$ https://www.indec.gob.ar/bajarPublicacion.sp?idc=3E382994AA6E1CDFDC94787B24D143A1180C0FB15CC55A2AB4D7B2036C899A7D39343DDB3C15E3BF).

Censo Nacional de Población, Hogares y Viviendas 2010: censo del Bicentenario: resultados definitivos. Buenos Aires, 2012.

MONTEIRO, M. G. Alcohol y salud pública en las Américas. Un caso para la acción. Washington, DC, 2007.

NACIONES UNIDAS. Coverage of birth and death registration. New York, 2008. Disponivel em: 〈https://unstats.un.org/unsd/demographic-social/crvs/documents/Website_final_coverage. xls>. Acceso em: 10 jan. 2017.

NAGHAVI, M. et al. Algorithms for enhancing public health utility of national causes-of-death data. Population Health Metrics, v. 8, n. 9, 2010.

REHM, J.; PATRA, J.; BALIUNAS, D.; POPOVA, S.; ROERECKE, M.; TAYLOR, B. Alcohol consumption and the global burden of disease 2002. Ginebra: OMS, 2006.

RIBOTTA, B. Evaluación de la exactitud de los datos sobre la causa básica de muerte en América Latina. Revista Peruana de Epidemiología, v. 18, n. 3, p. 1-9, 2014.

RIDOLFO, B.; STEVENSON, C. The quantification of drug-caused morbidity and mortality in Australia 1998. Canverra: Australian Institute of Health and Welfare, 2001.

SEDRONAR. Estudio nacional sobre consumo de sustancias psicoactivas en población de 12 a 65 años de edad. Argentina: Secretaría de Programación para la Prevención de la Drogadicción y Narcotráfico, 2010.

WHO - World Health Organization. Global status report on alcohol and health-2014. Luxembourg, 2014. 


\title{
Sobre los autores
}

Carola Leticia Bertone es licenciada en Kinesiología y Fisioterapia, Médica, magister y Dra. en Demografía. Investigadora de la Universidad Nacional de la Rioja (UNLAR) en el Centro de Estudios e Innovacion Tecnológica (CENIIT), en el Instituto de Demografia.

Víctor Eduardo Torres es licenciado en Economía, magister en Administración de Negocios y Dr. en Demografía. Docente de la Universidad Nacional de Córdoba (UNC) e investigador del Consejo Nacional de Investigaciones Cientificas y Técnicas (CONICET).

Marcos Javier Andrada es licenciado en Psicopedagogía, magister y Dr. en Demografía. Docente de la Universidad Nacional de La Rioja (CENIIT-UNLAR), en el Instituto de Demografia e investigador del Consejo Nacional de Investigaciones Cientificas y Técnicas (CONICET).

\section{Dirección para correspondencia}

\author{
Carola Leticia Bertone \\ Manzana, 1275, casa 23, Barrio Nestor Kirchner \\ 5300 - La Rioja, La Rioja, Argentina \\ Víctor Eduardo Torres \\ Vietes, 385, Barrio Alto Alberdi \\ 5003 - Córdoba, Córdoba, Argentina \\ Marcos Javier Andrada \\ Peatonal, 207, casa 24, Barrio Hospital \\ 5300 - La Rioja, La Rioja, Argentina
}

\begin{abstract}
Mortality related to alcohol consumption and its impact on life expectancy of the Argentinian Northwestern population, 2011

Out of the deaths that occurred in 2012 worldwide, 5.9\% were caused by harmful consumption of alcohol (WHO, 2014). In Argentina, during 2008 there were 11,013 deaths attributed to alcohol consumption (3.6\% of total deaths in population) (ACOSTA, BERTONE e PELÁEZ, 2012). Alcohol consumption per capita by people aged 15 and over in Argentina remained at around 9.5 liters of pure alcohol (equivalent to 25 grams of alcohol per day), considering average figures for the years 2003-2005 and 2008-2010 (WHO, 2014). This quantitative, descriptive and cross-sectional study aims to analyze mortality attributable to alcohol consumption in the provinces of the Argentinian Northwest in 2011, and how this affects life expectancy of these populations. The methodologies proposed by the CDC (1990) - Mortality Attributable to Alcohol Consumption (MACA) and Years of Lost Life Expectancy (AEVP) have been applied (ARRIAGA, 1996). Data provided by DEIS (vital statistics) and INDEC (EnPreCoSP-2011 and population projections) have been used. This article seeks to provide updated information on the status of mortality attributable to alcohol consumption, which is a contribution to public health in order to assess the implementation of appropriate strategies.
\end{abstract}

Keywords: Mortality. Alcohol. Argentinian Northwestern population. Years of Lost Life Expectancy. 


\section{Resumo}

Mortalidade relacionada ao consumo de álcool e seu impacto na expectativa de vida da população do noroeste argentino, 2011

No mundo, 5,9\% das mortes ocorridas em 2012 deveram-se ao consumo nocivo de álcool (WHO, 2014). Na Argentina, durante 2008 houve 11.013 mortes atribuídas ao consumo de álcool (3,6\% do total de mortes na população) (ACOSTA; BERTONE; PELÁEZ, 21012). O consumo per capita de álcool de pessoas com 15 anos ou mais na Argentina ficou em cerca de 9,5 litros de álcool puro (equivalente a 25 gramas de álcool por dia), considerando a média dos períodos 2003-2005 e 2008-2010 (WHO, 2014). Este trabalho, quantitativo, transversal e descritivo, visa estudar a mortalidade atribuível ao consumo de álcool nas províncias do noroeste argentino, em 2011, e como isso afeta a expectativa de vida dessas populações. A metodologia utilizada é proposta pelo CDC (1990) - aplicação da mortalidade atribuída ao consumo de álcool (MACA) e anos de expectativa de vida perdidos (AEVP) (ARRIAGA, 1996). Os dados das estatísticas de DEIS (estatísticas vitais) e INDEC (EnPreCoSP-2011 e projeções populacionais) são usados. Espera-se que este trabalho forneça informações atualizadas sobre o status da mortalidade atribuível ao consumo de álcool, importantes em termos de saúde pública para avaliar a implementação de estratégias apropriadas.

Palabras-chave: Mortalidade. Álcool. Noroeste argentino. Anos de expectativa de vida perdidos.

Recebido para publicação em 01/12/2017 Aceito para publicação em 21/06/2018 